\title{
Research and Design of Warehouse Environment and Personal Radiation Monitoring Experiment System
}

\author{
Li Biao*1, Wu Fei $^{1}$, Guo Ming ${ }^{1}$, Huang huang ${ }^{1}$ and Huang Gui ${ }^{1}$, Xu Zijian ${ }^{1}$ \\ ${ }^{1}$ College of Nuclear Science and Technology, Naval University of Engineering, Wuhan, Hu Bei, China
}

\begin{abstract}
With reference to the technical standards of radiation monitoring professionals in the "National Post and Occupational Skills Appraisal and Evaluation System", the idea of simulation system development and integration is adopted to build a warehouse environment and personal radiation monitoring experiment system for principle teaching and installation operations. Through the establishment of experiments such as environmental radiation dose measurement, surface pollution measurement and removal, personal dose measurement and alarm, radiation protection and emergency treatment, the students' theoretical knowledge has been consolidated and practical ability has been improved.
\end{abstract}

\section{Introduction}

With the development of nuclear-related equipment, higher requirements are placed on the practical ability of the team for radiation monitoring and protection ${ }^{[1-3]}$. After several rounds of course teaching, field lectures and field investigations on the major of radiation monitoring and protection, based on clarifying the capacity requirements of the profession, the construction of warehouse environment and personal radiation monitoring experiment system are carried out from the aspects of scientific top-level design, clear technical routes, and key technology research ${ }^{[4,5]}$.

\section{OVERALL DESIGN OF THE EXPERIMENT SYSTEM}

The warehouse environment and personal radiation monitoring experiment system includes four parts ${ }^{[6-8]}$ : environmental radiation monitoring equipment, personal dose monitoring equipment, simulated radiation detection training equipment and radiation protection equipment. The specific composition is shown in Figure 1.

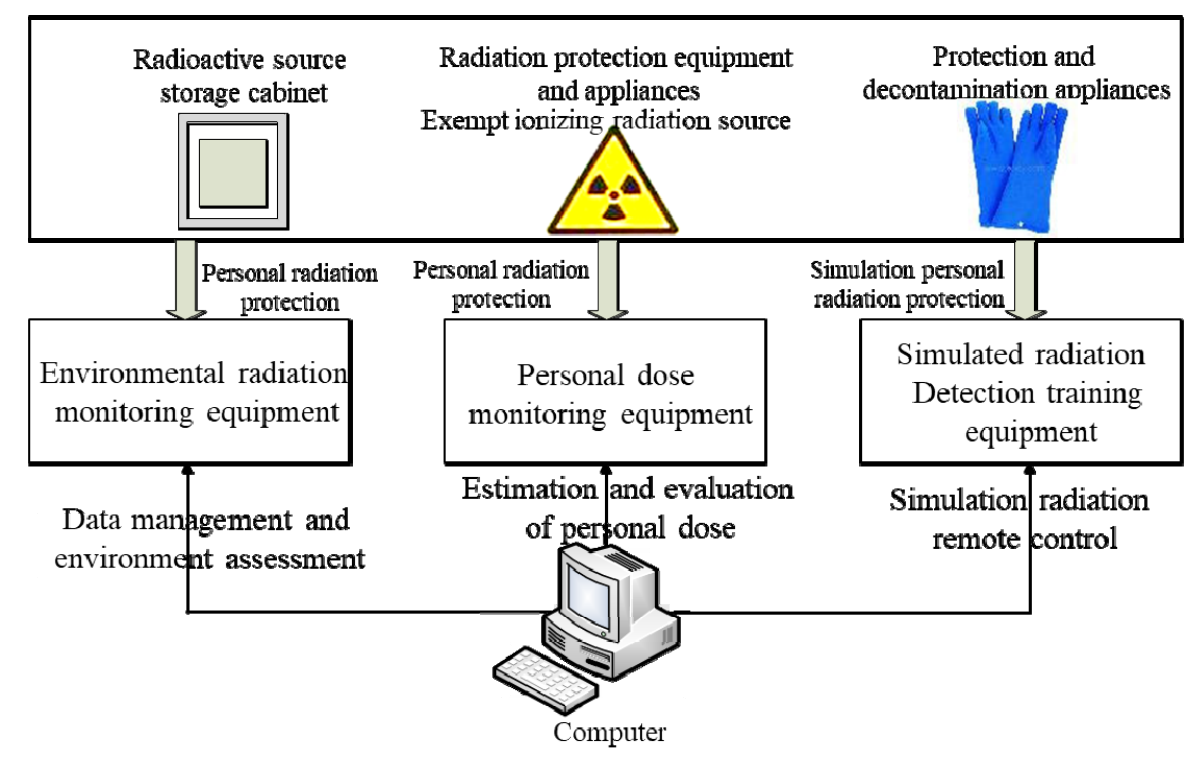

Fig1. Overall design of the warehouse environment and personal radiation monitoring experiment system

Environment radiation monitoring equipment includes

\subsection{Environment radiation monitoring equipment}

\footnotetext{
* Corresponding author: zjs19781129@,163.com
} dose monitoring equipment, activity measurement equipment and $\gamma$ spectrometer, including $\gamma$ dose rate meter, surface pollution detector, $\mathrm{NaI}(\mathrm{Tl})$ scintillation probe, 
multi-channel energy spectrum analysis system, oscilloscope, standard sample preparation equipment,
UPS power, etc. The composition of this type of equipment is shown in Figure 2.

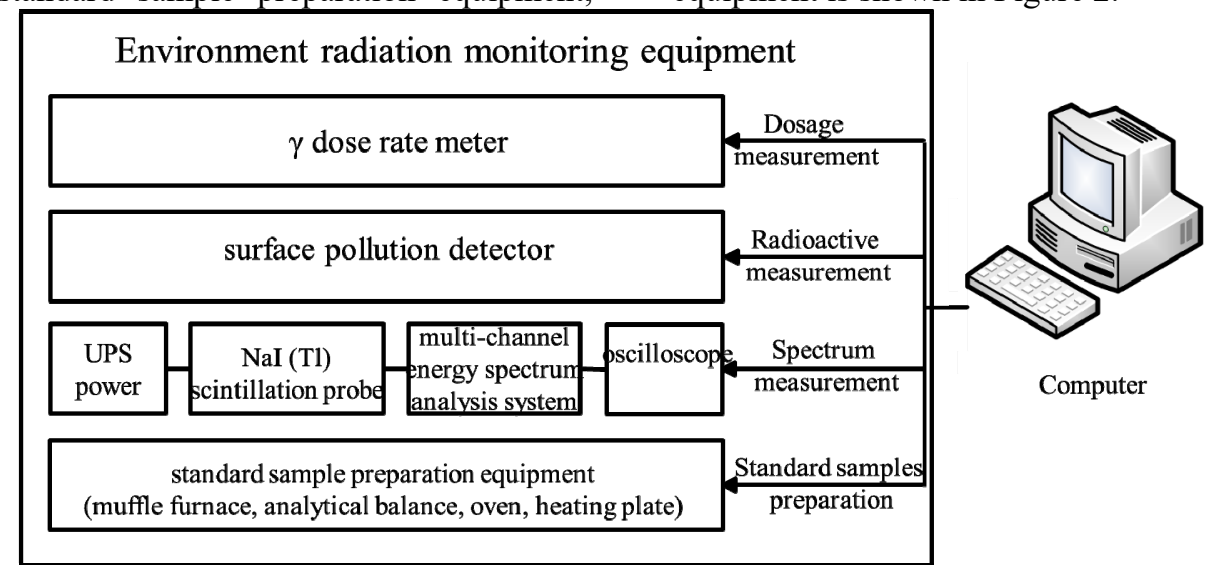

This type of equipment supports detection experiments of environmental background and low-level radioactivity, and promotes students to master the principles and techniques of radiation detection. The experimental items that can be carried out are as follows:

(1) Environmental radiation dose measurement experiment: The measurement and evaluation of environmental and abnormal $\gamma$ radiation are highly versatile and widely used in the fields of environmental monitoring inside and outside of the workplace, radiation protection, and various types of accident emergency;

(2) Workplace surface contamination detection experiment: Detect $\alpha$ and $\beta$ radioactivity on all surfaces (ground, desktop, cabinet, human body surface, etc.) in the workplace, and control harmful pollution areas and levels.

(3) Standard sample preparation experiment: Collect natural samples at workplace and surrounding environment, prepare standard samples for energy spectrum measurement and low-level radioactivity measurement experiments by physical and chemical methods in accordance with relevant national standards.

(4) Standard sample $\gamma$ spectrum measurement experiment: Use $\mathrm{NaI}(\mathrm{Tl}) \gamma$ spectrometer to measure and analyze the types and activities of radionuclides. While satisfying the experiment teaching of professional courses, it can perform radionuclide analysis tasks in routine monitoring, environmental assessment, safety analysis, emergency treatment, and site restoration.

\subsection{Personal dose monitoring equipment}

Personal dose monitoring equipment refers to intelligent personal dose alarm equipment, as shown in Figure 3.

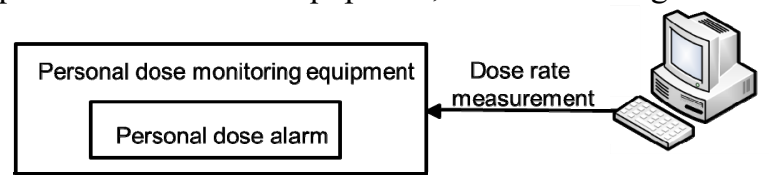

Fig3. Personal dose monitoring equipment
The device supports personal dose monitoring experiments of equipment operators and radiation monitors, and promotes students to establish correct and scientific radiation protection awareness and skills. And can implement personal dose rate measurement and alarm implementation: master the use of direct-reading electronic dose alarm equipment, set alarm thresholds reasonably, and clarify the compensation relationship between the alarm device and the thermoluminescence dosimeter.

\subsection{Radiation detection simulation training equipment}

Nuclear material is highly radioactive and chemically toxic, and its physical properties make it easily form diffuse pollution to the operating site ${ }^{[9]}$. The country has imposed strict controls on nuclear materials. It is difficult for laboratories to obtain nuclear materials, and some tunnel emergency monitoring and disposal operations for nuclear materials are difficult to carry out in laboratories ${ }^{[10,11]}$. Therefore, in order to solve the actual operation of above sensitive substances based on the existing conditions, and restore the actual status of the post truly, simulated radiation sources and detection equipment for nuclear material surface pollution and radiation field detection are developed to support related implementations in teaching and training. This type of simulation training equipment includes two types of simulated radiation sources, two types of simulated radiation detectors, and simulated pollutants. The specific composition is shown in Figure 4.

This simulation training system can support the following implementations:

(1) Low-level surface contamination removal practice: Skillfully use various surface decontamination methods and implement the low-level surface contamination removal implementation in accordance with the "detection-decontamination-detection" process. 


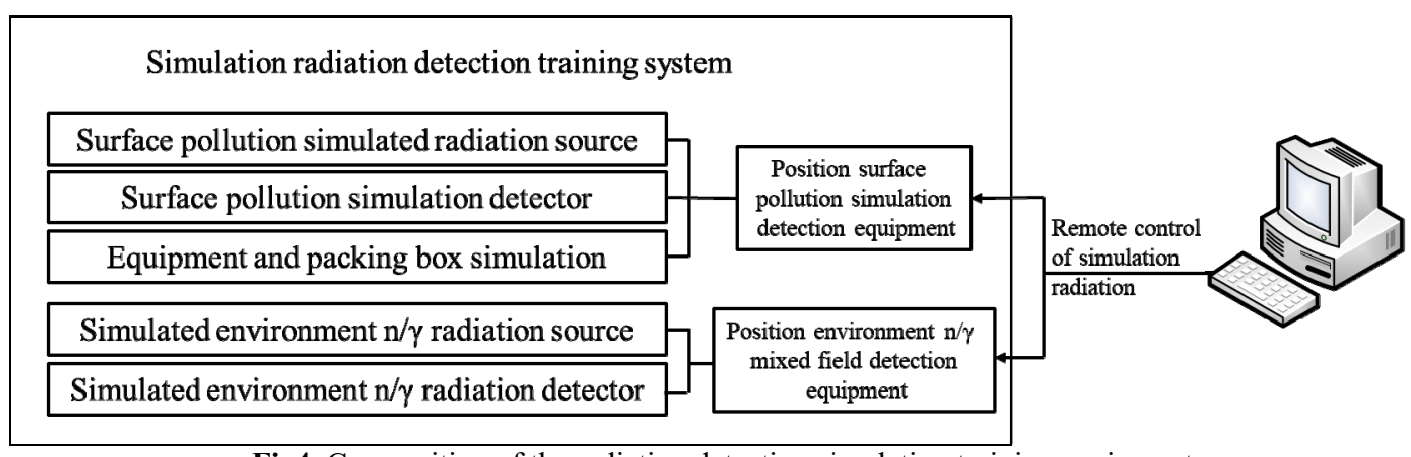

Fig4. Composition of the radiation detection simulation training equipment

(2) Emergency monitoring and disposal drill for equipment operation in the tunnel: including surface pollution ( $\alpha, \beta$ pollution on horizontal surface) detection simulation implementation, environment neutron $/ \gamma$ mixed radiation field simulation survey implementation and packaging box pollution (packing box surface $\alpha, \beta$ pollution and $\alpha$ aerosol concentration in the air in the container) Simulated detection and other practical content, using simulated radiation field, simulated detection equipment and protective equipment to complete the emergency monitoring and disposal of some tunnels in the equipment operation.

(3) Implementation of emergency monitoring of radiation pollution accidents: Organically combining the relevant equipment of the simulation training system, the environmental radiation monitoring system and the personal dose monitoring system to realize the radiation emergency monitoring and personal protection operations under conditions of nuclear events and accidents.

\subsection{Radiation protection equipment and appliances}

In order to ensure personal and environmental safety in radiation monitoring experiments, and promote students to develop awareness of work and habits in radiation safety, various types of radiation protection equipment and appliances are constructed and procured, mainly including exempt ionizing radiation sources $(\alpha, \beta, \gamma$ sources)、 Standard storage facilities for radioactive sources, personal protective equipment, laboratory radioactive solid waste collection containers and decontamination appliances, and full life management of radioactive sources are implemented in accordance with relevant regulations of the Ministry of Environmental Protection and national standards. Since radiation safety is accompanied by all nuclear activities, all previous experiment implementations required the use of radiation protection equipment and appliances. In addition, in combination with personal dose monitoring experiments and simulated radiation monitoring operations, a project on the use of radiation protection appliances in the workplace is set up to promote students' knowledge and mastery of the use and scope of personal radiation protection appliances.

\section{TECHNICAL ROUTE OF EXPERIMENTAL SYSTEM CONSTRUCTION}

With the goal of cultivating students' skill of radiation monitoring and pollution control, according to radiation types and in-use equipment procedures, radiation monitoring equipment simulators are developed, environmental dose monitoring equipment and radiation protection equipment and appliances are purchased, and warehouse environment and personal radiation monitoring systems are set up.

\subsection{Development of simulation instruments}

\subsubsection{Design ideas for simulation instruments}

Simulation instruments include simulated radiation detection equipment and simulated pollutants. Simulated radiation detection equipment is divided into surface pollution simulation detection equipment and workplace environment neutron $/ \gamma$ mixed field detection equipment. Both devices use computer-controlled radio transmission to simulate the radiation source, and the computer remote control device can set the radiation type and radiation field distribution. After the simulation detector receives the wireless signal, it outputs the radiation dose after signal analysis ${ }^{[12-14]}$.Figure 5 is a schematic block diagram of an simulation radiation source and detection equipment.

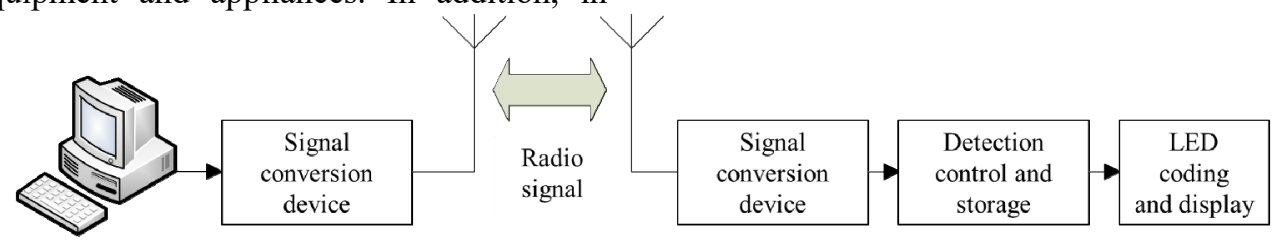

Fig5. Schematic block diagram of the simulation radiation source and detection equipment 


\subsubsection{Design of surface pollution simulation detection equipment}

In the surface pollution simulation detection equipment, metal pole plates are used to simulate pollution sources. Capacitance detectors are used in combination with signal acquisition, processing, and control modules to simulate pollution source detectors. The simulated detectors have built-in wireless receiving devices and can be remotely controlled by a computer to set up pollution detection mode. The entire system can complete the simulated measurement of surface contamination in accordance with installed operating procedures. The principle of the surface pollution simulation detector is shown in Figure 6.

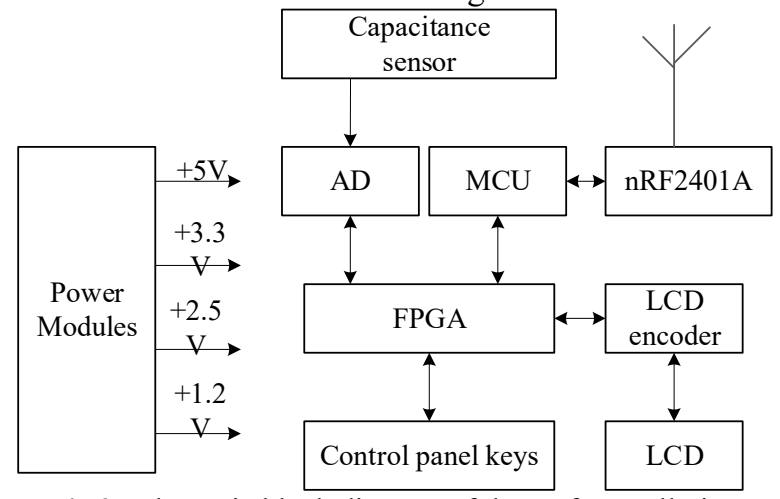

Fig6. Schematic block diagram of the surface pollution simulation detector

\subsubsection{Design of workplace environment $n / \mathrm{V}$ simulation detection equipment}

Workplace $\mathrm{n} / \gamma$ mixed field detection equipment uses wireless transmitting device to simultaneously simulate neutron and $\gamma$ two kinds of radiation. Through changing the device's location manually and computer-configured radiation coding to complete the radiation field simulation of the operating room (laboratory). A simulation detector with a receiving probe is installed to complete the environmental radiation field survey. Figure 7 illustrates the corresponding detector schematic.

The appearance of all simulated detectors is consistent with the army's radiation monitoring equipment on duty to achieve seamless docking with the army's actual operations. Except for the functional chip inside the simulation detector, the rest of the electronic system and configuration are consistent with the current mainstream surface pollution detection equipment to support the teaching of structural principles.

In order to fully simulate the radiation detection and decontamination process, each simulated radiation experiment needs to perform functional simulation of the input and output response of the detection instrument to achieve the dynamic setting of the measurement function and the dynamic display of measurement data. Therefore, it is necessary to develop supporting software for test and control in combination with simulation radiation detection equipment. The software is designed using FPGA-based Verilog language, which mainly includes data acquisition and processing, test mode control, LCD display control and other modules.

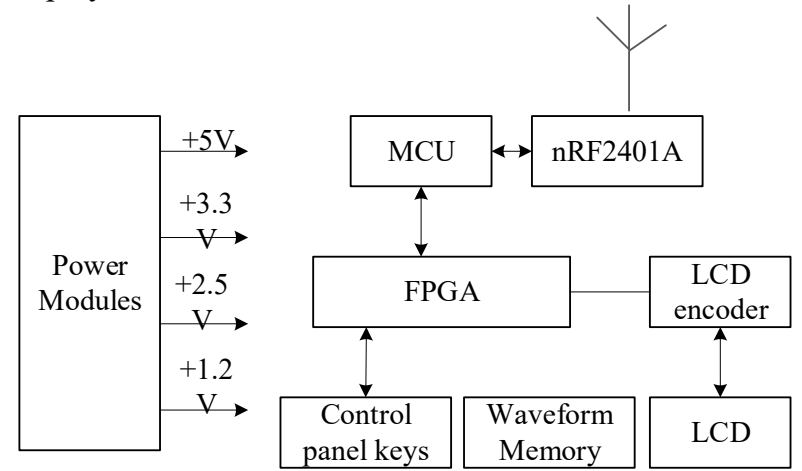

Fig7. Schematic block diagram of the $\mathrm{n} / \gamma$ simulation detection equipment

\subsection{Equipment selection}

The laboratory equipment that needs to be purchased for laboratory construction includes some monitoring equipment and protective equipment, mainly including $\gamma$ dosimeters, surface contamination detectors, $\mathrm{NaI}$ (Tl) scintillation probes, multi-channel analysis spectrum systems, spectrometer computers, oscilloscopes, UPS power supplies, standard sample preparation equipment, personal dose alarms, standard storage facilities for radioactive sources, waste collection containers and protective equipment, etc.

\subsection{EXPERIMENTAL SYSTEM INTEGRATION}

The implementation of the warehouse environment and personal radiation monitoring system depends on the debugging and function development between various equipment. During the construction of the experiment system, the overall plan of "functional analysis, requirements determination, overall design, resource acquisition, detailed design, system testing, system application, application evaluation" was adopted and started with experimental function for designing the laboratory in modules that can integrate with the whole, and completed the construction of the laboratory through systematic testing, application and evaluation. Due to the particularity of the hazardous substances involved in ionizing radiation, the laboratory layout needs to be partitioned and graded in accordance with relevant regulations and standards to ensure the safety of personnel and equipment.

There are two types of experiment systems involving nuclear operations. Radiation monitoring experiments generally involve the use of radioactive materials and need to be managed in zones in accordance with relevant national regulations strictly. Therefore, it is intended to be divided into radiation monitoring experimental areas. The simulation equipment does not use radioactive sources, and only uses a computer to simulate the radiation field. Therefore, a simulation detection operation area can be set up separately. In addition, for the safety management of exempt radioactive sources, various types of wastes and small amounts of chemical 
reagents, a dangerous goods storage room was set up independently and constructed in accordance with relevant national standards.

\section{KEY TECHNOLOGIES OF EXPERIMENTAL SYSTEM CONSTRUCTION}

\subsection{Radiation source simulation and measurement technology}

Surface pollution simulation detection equipment and workplace environment $\mathrm{n} / \gamma$ mixed field detection equipment are simulated with surface alpha and beta pollution from nuclear materials and environmental $n / \gamma$ radiation from neutron sources, respectively. The spatial distribution of two types of radiation is very different, resulting in different technical methods used in the simulation of the radiation source. Different designs are used in the specific development process, and the detection and control channels that accompany and distinguish between each other are developed. The use of radio signals to simulate the detection of four kinds of rays and integrate them into an experimental system is difficult to develop, but has a broad application prospect.

The solution of this technical difficulty depends on the system design and experiment exploration. This article adopts a two-way scheme based on capacitance sensor-based simulation radiation and digital codecontrolled radio simulation radiation field. The radiocontrolled detector sensitivity and computer-configured radiation code simulated radiation field are used in this paper.

\subsection{Nal (TI) y spectrometer system experiment technology}

At present, the whole $\gamma$ spectrometer machine is expensive, and the maintenance and debugging costs are high. Therefore, the method of purchasing key components and building the whole machine by self is adopted in the laboratory construction. Due to the high precision of the gamma spectrometer, the electronics requirements of different probes vary greatly, and the construction technology is difficult. The applicability of multichannel analysis software to specific nuclear materials has also been developed. After the system is set up, it is necessary to carry out long-term debugging, and regularly perform work such as spectrum stabilization, spectral drift, calibration, etc. The experiment technology is difficult, and referring to the requirements of the special nuclear material spectrum measurement technology on the spectrometer, a useful attempt has been made and development.

\section{CONCLUDES}

After the experiment system was completed, experiments such as environmental gamma radiation dose measurement, workplace surface pollution detection, personal dose rate measurement and alarm, standard sample preparation, gamma energy spectrum measurement and other experiments were conducted. Use of radiation protection appliances, emergency monitoring of radiation pollution accidents, and lowlevel surfaces pollution removal and equipment operation emergency monitoring and disposal drills in the tunnel and other practical projects meet the needs of professional course teaching and practical implementation. The experiment system can be used as a research platform for related technologies in the radiation monitoring specialty of the warehouse, and it can also provide a basic skill training platform for onthe-job technical backbone training of related majors to strengthen the effect of on-the-job education.

\section{References}

1. Lei Bin, Cai Junfeng. Exploration and thinking on construction of laboratories in military academies[J]. Experiment Technology and Management, 2012, 29(10): 191-193.

2. Liu Tuo, Qu Bo. Innovation of laboratory construction and talent cultivation system[J]. Experiment Technology and Management,2009, 26(4): 237-240.

3. Luo Zhengxiang. Study and practice of laboratory construction for application-type talents cultivation in independent college[J]. Experiment Technology and Management, 2011, 28(7). 1-5.

4. Hu Bita, Zhang Yu. Construction of nuclear science and technology experiment teaching system [J]. Higher Education of Sciences, 2009,(4): 100-102.

5. Lu Dianqing, Li Xinhua, Cai Li, Shi Linxing, Shao Litang, Liu Xuedong. Exploration and practice on specialized laboratory construction of new energy science and engineering of application-oriented universities $[\mathrm{J}]$. Experiment Technology and Management, 2014, 31(12): 226-229.

6. Li Huafang. Introduction to electromagnetic radiation protection technology and research laboratory [J]. Safety, 2011,(6): 55-56.

7. Yang Qiyong. Exploration and practice of chemical laboratory construction of newly founded undergraduate colleges and universities[J]. Experiment Technology and Management, 2012, 29(10): 193-196.

8. Zhang Zhiqiang, Li Enjing, He Ping. Design and implementation of management system for radiation protection in Peking University[J]. Experiment Technology and Management, 2012, 29(3): 106-108.

9. Yang Xiaohe, Zhu Wencai. Workplace Radiation monitoring and nuclear safety radiation protection management in workplace [J]. Annual Report of Chinese Academy of Atomic Energy, 2001: 124-125

10. Xiao Lin. Analysis on Safety Management of Laboratory Radioactive Sources [J]. Industrial Health and Occupational Diseases, 2007, 33(2): 102-104. 
11. Li Xueqin, Dang Lei, Cao Jisheng, Zhang Wijun. Discussion on Radiation Safety and Protection Management in a Laboratory[J]. China Occupational Medicine,2012, 39(1): 68-40.

12. Chen Shihai, Wang Enyuan, Shi Xiankui, Hao Yankun. Mobile electromagnetic radiation of coal or rock monitoring system $[\mathrm{J}]$. Journal of China Coal Society, 2011, 36(S1): 137-141

13. Shen Ming, Zhu Yuelong, Zhao Yanzi. The Issue of Gamma Spectral System SourcelessObject Calibration Software Using in Radioactive Environment Measurement [J]. Nuclear Electronics and Detection Technology, 2009, 29(1): 116-112.

14. Chen Mingchi, Zhou Sichun, Wang Pan, Wang Xiaoqiong. Design of Wireless Radiation Detection Alarm Baed on ZigBee Technology [J]. Nuclear Electronics and Detection Technology, 2009, 29(3): 697-700. 